\title{
Semi classical chaos in a 2-D exactly solvable system
}

\author{
A. Nanayakkara* \\ Institute of Fundamental Studies, Hanthana Road, Kandy
}

\begin{abstract}
The Chaotic behavior of the classical trajectories and the level spacing distributions of 2-D Pseudo Hermitian Hamiltonian $H=\frac{1}{2}\left(p_{x}{ }^{2}+p_{y}{ }^{2}\right)+\frac{1}{2}\left(\omega_{x}^{2} x^{2}+\omega_{y}^{2} y^{2}\right)-i g x y$, where $g$ is a real parameter are investigated. This Hamiltonian is separable and Lyapunov exponents are obtained analytically. All the trajectories of this system are found to be regular and Hamiltonian is Hermitian when $\frac{\left|\omega_{x}^{2}-\omega_{y}^{2}\right|}{2} \geq|g|$ and trajectories become chaotic and Hamiltonian becomes Pseudo Hermitian when $\frac{\left|\omega_{x}^{2}-\omega_{y}^{2}\right|}{2}<|g|$. The level spacing distributions are obtained for both real and complex eigen energies. It was interesting to find out that level spacing distributions of complex norm of the eigen energies which are corresponding to chaotic motion show Poisson like distribution rather than Gaussian ensamble statistics.
\end{abstract}

\section{INTRODUCTION}

Most common way of determining classical chaos is the exponential divergence in time of neighboring points in phase space. The quantitative measure of the degree of this irregular/chaotic behavior is provided by the Lyapunov exponents which give, roughly speaking, the rate of exponential divergence of nearby trajectories ${ }^{1}$.

On the other hand, indicator most commonly used in quantum chaos is the statistical quantum properties of systems within the framework of random matrix theory

\footnotetext{
* Corresponding author
} 
(RMT). It is well known ${ }^{2,3}$ that the energy level statistics of quantum systems show different statistical characteristics corresponding to whether the system is chaotic or regular in the classical limit ${ }^{4}$. For systems which are regular in the classical limit, the energy levels correspond to Poisson statistics, while for systems which are chaotic in the classical limit, the statistics follow Gaussian ensemble statistics or Wigner statistics according to the system's symmetry. Most of the Hamiltonian systems which have been studied in the literature are Hermitian and hence entire quantum spectra of them are real.

$P T$-symmetric, non Hermitian, complex Hamiltonians are found to possess real quantal spectra even in higher dimensions ${ }^{5}$. Also it has been found that classical trajectories of these $P T$-symmetric Hamiltonians in complex phase space show regular behavior at low energies while chaotic behavior at high energies ${ }^{6}$.

Recently, Mostafazadeh, ${ }^{7-9}$ has generalized $P T$-symmetry by pseudo-Hermiticity and showed that eigenvalues of pseudo-Hermitian Hamiltonians are either real or they come in complex conjugate pairs. A Hamiltonian $H$ is said to be $\eta$ - pseudo-Hermitian if $H^{+}=\eta H \eta^{-1}$ where $^{+}$denotes their adjoint operator and $\eta$ is a Hermitian invertible linear operator. The complex Hamiltonians with real spectra are specially interesting as they can be generalized to quantum field theories. A number of such generalization s have recently been examined $5,10,11$.

\section{2-D PT SYMMETRIC SYSTEMS}

In this paper we report results of our study on the chaotic behavior of the system $H=\frac{1}{2}\left(p_{x}{ }^{2}+p_{y}{ }^{2}\right)+\frac{1}{2}\left(\omega_{x}^{2} x^{2}+\omega_{y}^{2} y^{2}\right)-i g x y$

Studying of the above system is important to the field of quantum chaos due to two main reasons. Firstly, investigations, such as this, will enable us to find out how the well known connections between semiclassical chaos and statistical behavior of quantum spectra changes when the systems are not Hermitian. Consequently, it provides clues to have a better understanding of the mechanism which connects semiclassical chaos with statistical properties of the corresponding quantum system. Secondly, since this system is separable and hence exactly solvable, one can have an analytic insight into the global behavior of the system.

In this study, we obtained expressions for classical trajectories in complex phase space and calculated Lyapunov exponents analytically to identify classical chaos in the system. Then we show that quantum level spacing distributions correspond to classical chaotic energies obey Poisson's distributions rather than usual Wigner type distributions (Gaussian ensemble statistics). 
The system in (1) is exactly solvable. It is not $P T$ symmetric but $\eta$-pseudoHermitian. It was found that ${ }^{12}$, when $\frac{\left|\omega_{x}^{2}-\omega_{y}^{2}\right|}{2} \geq|g|$ the entire spectra of this system is real and when $\frac{\left|\omega_{x}^{2}-\omega_{y}^{2}\right|}{2}<|g|$, some eigenvalues are real and others are complex and come as conjugate pairs. Also it was found that this system is Hermitian when $\frac{\left|\omega_{x}^{2}-\omega_{y}^{2}\right|}{2} \geq|g|$ and it becomes $\eta$-pseudo Hermitian when $\frac{\left|\omega_{x}^{2}-\omega_{y}^{2}\right|}{2}<|g|$, where $\eta$ now represents the exchange operator. In order to study this system classically, first we transform Hamiltonian $H$ into a separable form.

$H=\frac{p_{X}^{2}}{2 m_{X}}+\frac{p_{Y}^{2}}{2 m_{Y}}+\frac{1}{2} m_{X} \mu_{X}^{2} X^{2}+\frac{1}{2} m_{Y} \mu_{Y}^{2} Y^{2}$

where

$\mu_{X}=\sqrt{\frac{1}{2}\left[\left(\omega_{x}^{2}+\omega_{y}^{2}\right)-\sqrt{\left(\left(\omega_{x}^{2}-\omega_{y}^{2}\right)^{2}-4 g^{2}\right.}\right]}$

and

$$
\begin{aligned}
& \mu_{Y}=\sqrt{\frac{1}{2}\left[\left(\omega_{x}^{2}+\omega_{y}^{2}\right)+\sqrt{\left(\left(\omega_{x}^{2}-\omega_{y}^{2}\right)^{2}-4 g^{2}\right.}\right]} \\
& X=\lambda_{1} x+y, Y=\lambda_{2} x+y, p_{X}=\frac{p_{x}-\lambda_{2} p_{y}}{\lambda_{1}-\lambda_{2}}, p_{Y}=\frac{p_{x}-\lambda_{1} p_{y}}{\lambda_{1}-\lambda_{2}} \\
& \lambda_{1}=-\frac{\left[\left(\omega_{x}^{2}-\omega_{y}^{2}\right)-\sqrt{\left(\left(\omega_{x}^{2}-\omega_{y}^{2}\right)^{2}-4 g^{2}\right.}\right]}{2 i g} \\
& \lambda_{2}=-\frac{\left[\left(\omega_{x}^{2}-\omega_{y}^{2}\right)+\sqrt{\left(\left(\omega_{x}^{2}-\omega_{y}^{2}\right)^{2}-4 g^{2}\right.}\right]}{2 i g} . \text { and } m_{X}=\frac{1}{\left(1+\lambda_{1}^{2}\right)}, \quad m_{Y}=\frac{1}{\left(1+\lambda_{2}^{2}\right)}
\end{aligned}
$$

Quantum eigenvalues of (2) are given by;

$$
E_{n_{1}, n_{2}}=\left[n_{1}+\frac{1}{2}\right] \mu_{1}+\left[n_{2}+\frac{1}{2}\right] \mu_{2} \text {. }
$$

Now when $\frac{\left|\omega_{x}^{2}-\omega_{y}^{2}\right|}{2} \geq|g|, \quad \mu_{1}$ and $\mu_{2}$ are both real and hence for all $n_{1}$ and $n_{2} E_{n_{1}, n_{2}}$ is real.

Therefore entire spectrum is real.

In this case, classically, the system represents a 2-D uncoupled Harmonic oscillator and hence,

$$
X(t)=A_{X} \sin \left(\mu_{X} t+\phi_{X}\right), \quad p_{X}(t)=A_{X} m_{X} \mu_{X} \cos \left(\mu_{X} t+\phi_{X}\right)
$$


$Y(t)=A_{Y} \sin \left(\mu_{Y} t+\phi_{Y}\right), \quad p_{Y}(t)=A_{Y} m_{Y} \mu_{Y} \cos \left(\mu_{Y} t+\phi_{Y}\right)$

and motion is regular for all energies.

However, when $\frac{\left|\omega_{x}^{2}-\omega_{y}^{2}\right|}{2}<|g|, \mu_{X}$ and $\mu_{Y}$ become complex and $\mu_{X}=\bar{\mu}_{Y}=\bar{\mu}$.

$\mu=\sqrt{\frac{1}{2}\left[\left(\omega_{x}^{2}+\omega_{y}^{2}\right)-i \sqrt{4 g^{2}-\left(\omega_{x}^{2}-\omega_{y}^{2}\right)^{2}}\right]}$ and $\bar{\lambda}_{2}=-\lambda_{1}=\hat{\lambda}$. Then $m=m_{X}=\bar{m}_{Y}=1+\hat{\lambda}^{2}$

with $\hat{\lambda}=\frac{\left[\left(\omega_{x}^{2}-\omega_{y}^{2}\right)-i \sqrt{\left(4 g^{2}-\left(\omega_{x}^{2}-\omega_{y}^{2}\right)^{2}\right.}\right]}{2 i g}$

Now Hamiltonian $H$ becomes

$H=\frac{p_{X}{ }^{2}}{2 m}+\frac{p_{Y}{ }^{2}}{2 \bar{m}}+\frac{1}{2} m \mu^{2} X^{2}+\frac{1}{2} \bar{m} \bar{\mu}^{2} Y^{2}$

Quantum eigenvalues of the above Hamiltonian are given by

$$
E_{n_{1}, n_{2}}=\left[n_{1}+\frac{1}{2}\right] \mu+\left[n_{2}+\frac{1}{2}\right] \bar{\mu}
$$

and have following properties. Bar represents the complex conjugation.

(1) The eigenenergy of the ground state is real; $\left(E_{0,0}=\frac{\mu+\bar{\mu}}{2}\right)$

(2) The eigenenergies of the states with $n_{1}=n_{2}$ are real.

(3) When $n_{1} \neq n_{2}$, eigenenergies come in conjugate pairs; $\left(E_{n_{1}, n_{2}}=\bar{E}_{n_{2}, n_{1}}\right)$

Now we study this case classically. Let $m=m_{r}+i m_{i}$ and $\mu=\mu_{r}+i \mu_{i}$. The phase space co-ordinates are now complex quantities and given by

$$
\begin{aligned}
& X(t)=A_{X} \sin \left(\mu t+\phi_{X}\right), \quad p_{X}(t)=A_{X} m \mu \cos \left(\mu t+\phi_{X}\right) \\
& Y(t)=A_{Y} \sin \left(\bar{\mu} t+\phi_{Y}\right), \quad p_{Y}(t)=A_{Y} \bar{m} \bar{\mu} \cos \left(\bar{\mu} t+\phi_{Y}\right)
\end{aligned}
$$

For large $t$

$$
\begin{aligned}
& X(t) \approx-\frac{\varsigma}{2 i} e^{\left|\mu_{i}\right| t} e^{-i \zeta\left(\mu_{r} t+\phi_{X}\right)} \quad p_{x}(t) \approx \frac{\mu \varsigma}{2} e^{\left|\mu_{i}\right| t} e^{-i \zeta\left(\mu_{r} t+\phi_{X}\right)} \\
& Y(t) \approx-\frac{\varsigma}{2 i} e^{\left|\mu_{i}\right| t} e^{i \zeta\left(\mu_{r} t+\phi_{Y}\right)} \quad p_{Y}(t) \approx \frac{\mu \varsigma}{2} e^{\left|\mu_{i}\right| t} e^{i \zeta\left(\mu_{r} t+\phi_{Y}\right)}
\end{aligned}
$$

Here we make constants $A_{X}=1$ and $A_{Y}=1$ and $\varsigma=\operatorname{sgn}\left(\mu_{i}\right)$ 


\section{LYAPUNOV EXPONENTS AND LEVEL SPACING DISTRIBUTIONS}

Lyapunov exponents define the rate of exponential divergence of nearby trajectories. We consider the trajectory $\gamma(t)$ starting at $t=0$ at the phase space point $\gamma(t=0)=\left(X, Y, p_{X}, p_{Y}\right)$. The stability of a trajectory with respect to changes in the initial conditions is expressed by the stability matrix $\mathbf{M}_{i, j}(t)=\frac{\partial \gamma_{i}(t)}{\partial \gamma_{j}(0)}$. The stability matrix depends on the time $t$ and on the phase space point $\gamma_{j}(0)$ which specifies the initial conditions of the trajectory $\gamma_{i}(t)$. Each phase space point $\gamma$ has its own stability matrix and hence its own set of Lyapunov exponents. From the stability matrix, Lyapunov exponent is defined as

$$
\lambda(t)=\lim _{t \rightarrow \infty} \frac{1}{t} \ln (\|\mathrm{M}(t)\|)
$$

where, for real phase space $\|$.$\| denotes the Euclidean norm. In complex phase space \gamma_{i}(t)$ 's are complex quantities and hence we introduce the complex norm for $\|$.$\| .$

By considering near by trajectories, we obtain one dimensional Lyapunov exponent $\lambda$ for the Hamiltonian (1) as $\lambda=\left|\mu_{i}\right|$ where $\mu_{i}$ is the imaginary part of $\mu$, given by $\mu_{i}=\sqrt{\frac{1}{2}\left[-\frac{\left(\omega_{x}^{2}+\omega_{y}^{2}\right)}{2}+\sqrt{g^{2}+\omega_{x}^{2} \omega_{y}^{2}}\right]}$. When $\frac{\left|\omega_{x}^{2}-\omega_{y}^{2}\right|}{2} \geq|g|, \quad \mu$ is real and Lyapunov exponent is zero for all energies. Therefore all trajectories are regular. However, when $\frac{\left|\omega_{x}^{2}-\omega_{y}^{2}\right|}{2}<|g|,\left|\mu_{i}\right|$ is positive and hence it is corresponding to irregular or chaotic motion. Hence phase transition, regular to chaos, occurs at $|g|=\frac{\left|\omega_{x}^{2}-\omega_{y}^{2}\right|}{2}$. At this transition, frequencies $\mu_{1}$ and $\mu_{2}$ are equal and $\mu_{1}=\mu_{2}=\sqrt{\frac{\omega_{x}^{2}+\omega_{y}^{2}}{2}}$ and $\mu_{i}=0$.

It is known that the classical chaos manifest itself in the statistical properties of the quantum spectrum, such as the distribution of the magnitude of spacing between adjacent eigenvalues. For real multidimensional systems the level spacing distribution of a completely regular system assumes a Poisson-like form ${ }^{4}$.

$$
P_{P}(S) \sim \frac{1}{\Delta} e^{-S / \Delta}
$$

where $\Delta$ is the mean spacing. A completely irregular system on the other hand shows Wigner like level spacing distributions (GOE, GUE or GSE) as found by many numerical calculations ${ }^{13,14}$. Distinction between two types of distributions have been confirmed for real eigenvalues corresponding to real potentials by many investigators. In this study we investigated whether the regular and irregular energy levels assume Poisson like and Wigner like distributions respectively even for non-Hermitian systems such as (1). 
However, when the system is classically chaotic, most of the corresponding eigen values are complex, therefore we studied the level spacing distribution of complex norm $\varepsilon$ and the phase angles $\theta$ of the energy levels, where energy is expressed as $E=\varepsilon e^{i \theta}$.

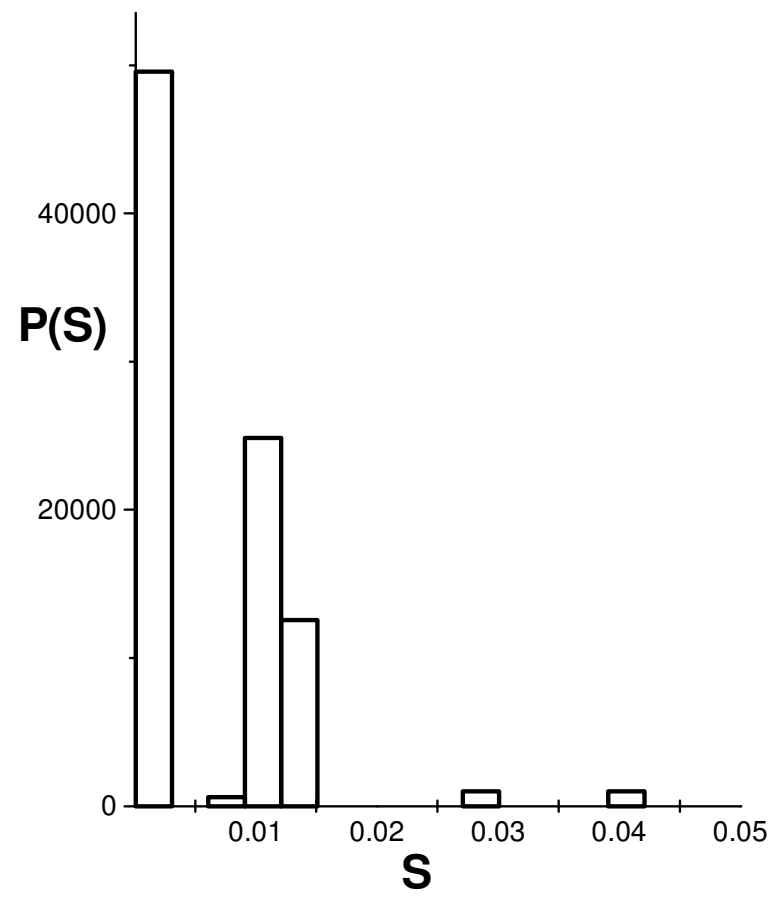

Figure 1a. Level spacing distribution of real energy $E$ correspond to classically regular notion. $\omega_{x}=0.7$, $\omega_{y}=1.3, g=-0.5$ and the Lyapunov exponent $\lambda=0$.

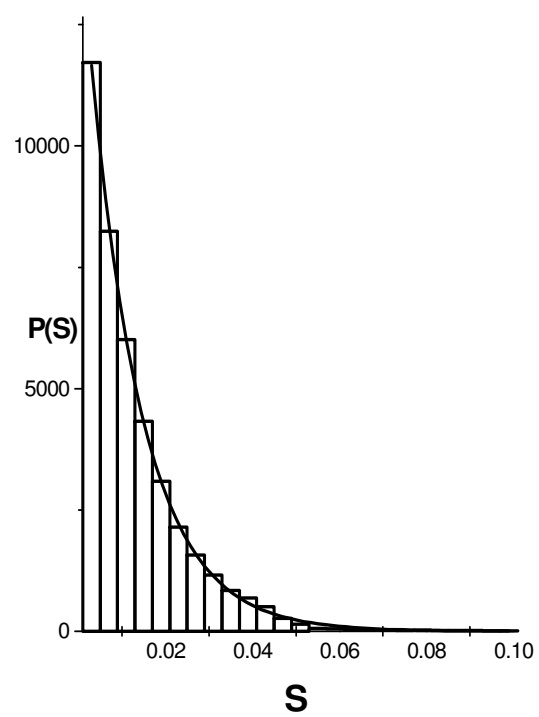

Figure 1b. Level spacing distribution of norm $(\varepsilon)$ of complex energy when the corresponding classical notion is chaotic. $\omega_{x}=0.7, \omega_{y}=1.3, g=-1.0$ and the Lyapunov exponent $\lambda=0.361989$. The curve is fitted to the histogram with Poisson distribution 


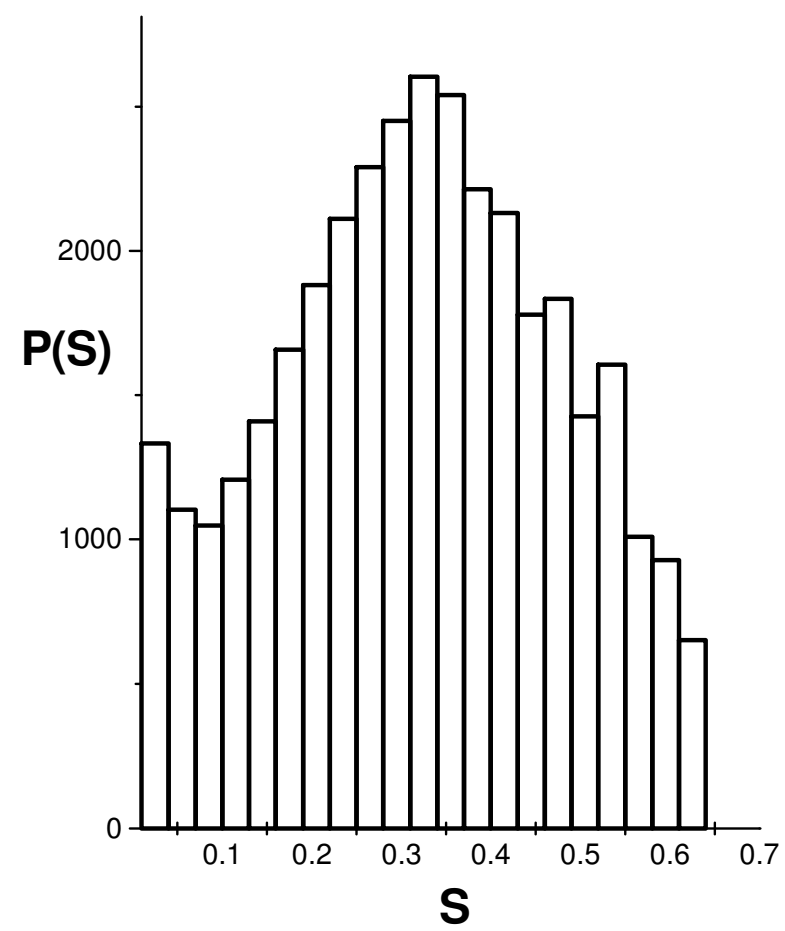

Figure 1c. For same parameters as in (b), the level spacing distribution of phase angle $(\theta)$ of complex energy.

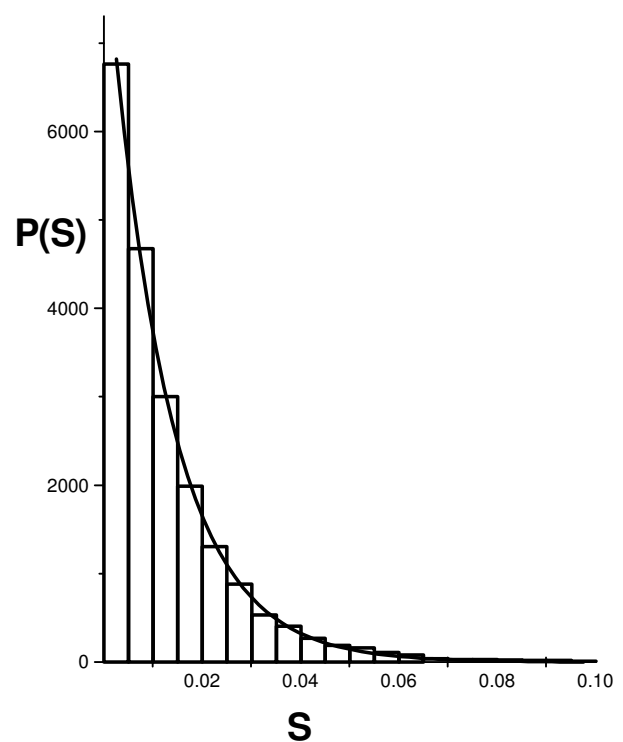

Figure 2a. This plot is for $\omega_{x}=1.0, \omega_{y}=1.0, g=-0.5$. When $\omega_{x}=\omega_{y}$ classical motion is chaotic for all values of $g$. The Lyapunov exponent for this case is $\lambda=0.242934$. 


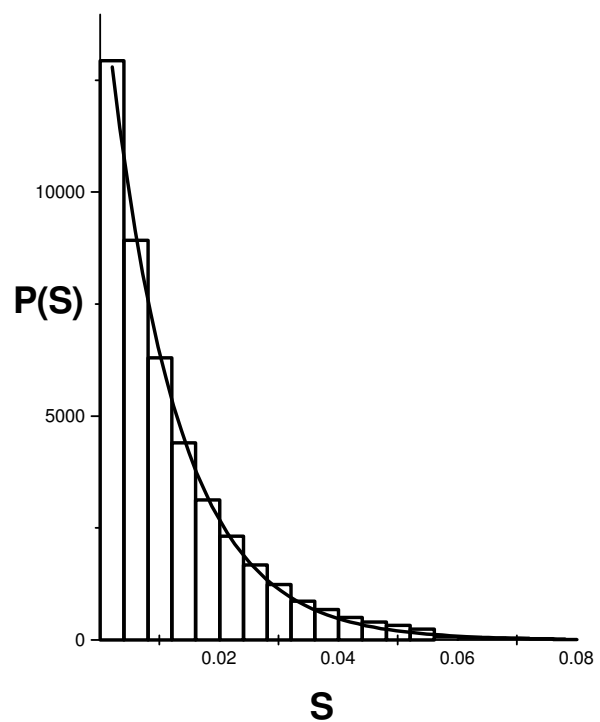

Figure 2b. Level spacing distribution of norm $(\mathcal{E})$ of complex energy when the corresponding classical motion is chaotic. $\omega_{x}=0.7, \omega_{y}=1.3, g=-1.0$ and the Lyapunov exponent $\lambda=0.45509$. The curve is fitted to the histogram with Poisson distribution

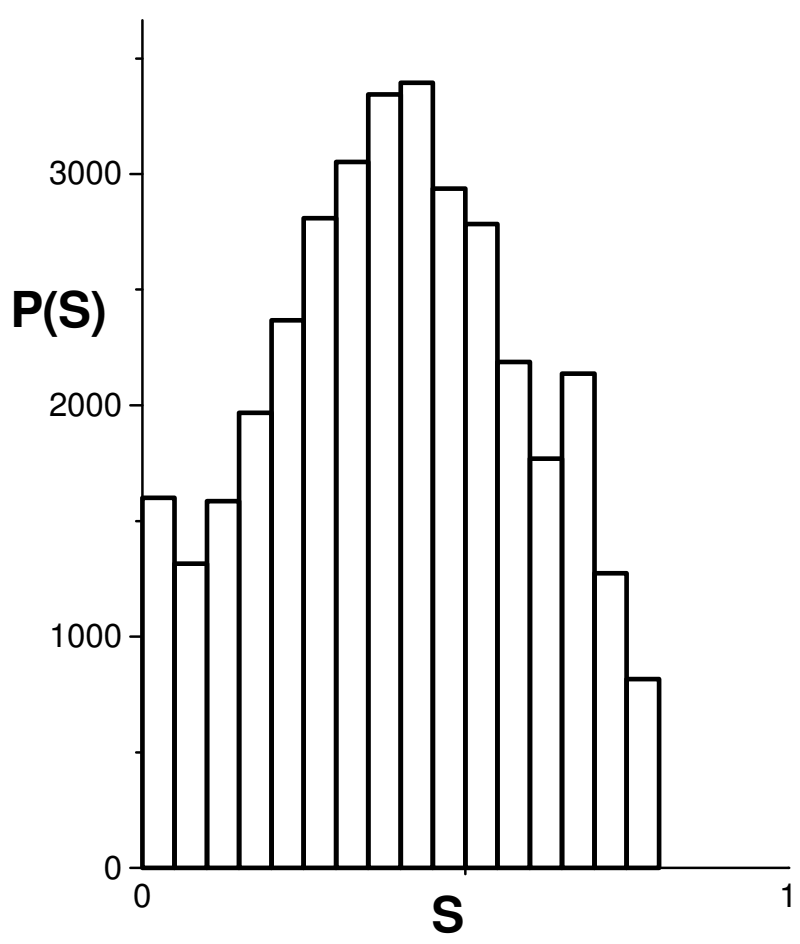

Figure 2c. For same parameters as in (b), the level spacing distribution of phase angle $(\boldsymbol{\theta})$ of complex energy. 
Figure 1 and Figure 2 show histograms of level spacing distributions of energy for two sets of parameters of the Hamiltonian in (1) as indicated. Figure 1a corresponds to classical regular motion and the entire quantum energy spectrum is real for this case. Figures $1 \mathrm{c}$ and Figures $2 \mathrm{c}$ are the level spacing distribution of the phase angles. It was observed that the width of the histogram changes with the parameter g. Also they do not have any tail on the either side as in the case of Gaussian type distributions.

It is evident from figure $1 \mathrm{~b}$ and figures $2 \mathrm{a}$ and $2 \mathrm{~b}$ that for the system studied here, the level spacing distributions of energy levels which correspond to classically chaotic motion do not have usual Gaussian ensemble statistics, but have Poisson like distributions instead. If the classical motion is categorized as chaotic when the classical Lyapunov exponent is positive, then for the system we have studied here has features very different from real Hamiltonian systems have been studied so far.

\section{REFERENCES}

1. H. D. Meyer, Fractals, Quasicrystals, Chaos, Knots and Algebraic Quantum Mechanics, (Kluwer Academic Publishers, 1988) p143.

2. L. E. Reichl, The Transition to Chaos in Conservative Classical systems; Quantum Manifestations (Springer-Verlag, Berlin, 1992)

3. M. L. Metha, Random Matrices (Academic Press, San-Diego 1991)

4. M. V. Berry and M. Tabor, Proc. Royal Soc. A 356, 375 (1977)

5. C. M. Bender, G. V. Dunne, P. N. Meisinger, M. Simsek, Phy. Lett. A, 311 (2001)

6. A. Nanayakkara and C. Abayaratne, Can. J. Phys., 81, 835(2003)

7. A. Mostafazadeh, J. Math. Phy. 43, 205 (2002)

8. A. Mostafazadeh, J. Math. Phy. 43, 2814 (2002)

9. A. Mostafazadeh, J. Math. Phy. 43, 3944 (2002)

10. C.M. Bender, S. Boettcher, P. N. Meisinger, J. Math. Phy. 40, 2201 (1999)

11. C. M. Bender, S. Boettcher, H. F. Jones, P. N. Meisinger, and M. Simsek, Phy. Lett. A, 291, 197 (2001)

12. A. Nanayakkara Phy. Lett. A, 304, 67 (2002)

13. S. W. McDonald and A. N. Kaufman, Phy. Rev. Lett. 42, 1182 (1979).

14. P. Pechukas, Phys. Rev. Lett. 51, 943 (1983) 